\title{
Impact Speed Does Not Determine Severity of Spinal Cord Injury in Mice with Fixed Impact Displacement
}

\author{
Joong Hee Kim,1 Tsang-Wei Tu, ${ }^{2}$ Philip V. Bayly, ${ }^{2,3}$ and Sheng-Kwei Song ${ }^{1}$
}

\begin{abstract}
The speed of three leading rodent SCI impacting devices- $0.1 \mathrm{~m} / \mathrm{s}$ (Infinite Horizon), $0.2 \mathrm{~m} / \mathrm{s}$ (Ohio State University), and $0.4 \mathrm{~m} / \mathrm{s}$ (New York University) - were investigated using a custom-fabricated impactor to determine its effect on mouse spinal cord injury severity. The spared white matter was examined at 7 and 21 days post-injury with in vivo diffusion tensor imaging (DTI) and post-mortem histology, respectively. The neurological outcome of the injured mice was longitudinally evaluated using the Basso mouse scale. In vivo DTI derived diffusion anisotropy maps provided excellent gray-white matter contrast enabling objective and noninvasive quantification of normal appearing white matter. In vivo DTI estimated spared white matter content correlated well with those determined using post-mortem histology. No significant difference in BMS was observed among injury groups of various impact speeds. The present results suggest that injury severity can be reproduced using speeds from 0.1 to $0.4 \mathrm{~m} / \mathrm{s}$ at the fixed impact displacement.
\end{abstract}

Key words: BMS; contusion SCI; diffusion tensor imaging; impact speed; spared white matter

\section{Introduction}

$\mathbf{T}$ He RODENT MODEL of contusion spinal cord injury (SCI) has been widely employed for preclinical studies and is considered an appropriate model of human SCI (Young, 2002). Contusion injury devices, driven by gravity or electromagnetic force, induce traumatic injury by rapid deformation of the spinal cord tissue. The contusion SCI model has been developed in rodents to mimic damages caused by vertebral dislocation or "burst" fracture. Producing consistent, controlled, and reproducible injury, essential for the successful application of an animal contusion SCI model, has been achieved by applying advanced technology (electronics, mechanics, computer software, etc.) in the design of injury devices (Rasband, 1997-2005; Stokes, 1992). Accurate monitoring of the mechanical parameters of impact by appropriate sensors reduces errors and enhances reproducibility of the performance of the impacting device.

The use of mice in SCI studies is common, in part because of the availability of transgenic and knock-out animals that provide valuable insight into the mechanism of pathogenesis and repair. Three major devices-the Ohio State University (OSU) (Rasband, 1997-2005; Jakeman et al., 2000); Infinite Horizon (IH) (Scheff et al., 2003); and the New York University (NYU) (Gruner, 1992; Kuhn and Wrathall, 1998)have been commonly used to generate controlled contusion
SCI in mice. All these devices provide measurements of biomechanical parameters, including force on the spinal cord, impact displacement, and velocity of the impact tip. To generate injuries with consistent severity, various approaches have been employed. The NYU device produces consistent injury severity by accurate control of the height of its impacting rod; this generates consistent impact speed, which, for targets of similar compliance, leads to consistent force and impact depth. The OSU device controls severity of injury by precisely regulating the impact displacement, the depth of the impact tip traveling from dura in the cord. The IH device achieves this goal by the real-time measurement and control of the force exerted on the cord.

The difficulty of producing consistent $\mathrm{SCI}$ injuries is partly attributed to the lack of a consensus identifying a single impact device that fits various research needs. One of the most distinct differences among the three major devices is the impact speed (or velocity) achieved at impact: $0.1 \mathrm{~m} / \mathrm{s}(\mathrm{IH})$, $0.2 \mathrm{~m} / \mathrm{s}(\mathrm{OSU})$, and $0.4 \mathrm{~m} / \mathrm{s}$ (NYU). Thus, the effect of the impact speed on the severity of the mouse SCI model should be investigated. Recently, a linear relationship between impact displacement and the force exerted on the spinal cord by the IH device has been reported (Ghasemlou et al., 2005). Motivated by these results, we evaluated the effect of impact speeds of $0.1,0.2$, and $0.4 \mathrm{~m} / \mathrm{s}$, with an accurately controlled displacement of $0.8 \mathrm{~mm}$.

${ }^{1}$ Department of Radiology, ${ }^{2}$ Department of Mechanical and Aerospace Engineering, and ${ }^{3}$ Department of Biomedical Engineering, Washington University, St. Louis, Missouri. 
Various methods have been used to evaluate the severity of SCI. Although histology is the "gold standard" for evaluating animal SCI severity, its invasive nature prohibits its application for longitudinal observations. Scoring of the animal's behavioral outcome is a relevant, noninvasive, and widely used metric (Basso et al., 1995, 1996, 2006; Engesser-Cesar et al., 2005). But this method lacks morphological and pathophysiological information on contusion-injured cords. For a better insight into the underlying injury, magnetic resonance imaging (MRI) has been employed as a noninvasive method to examine contusion-injured rodent spinal cord both ex vivo (Nevo et al., 2001; Schwartz and Hackney, 2003; Schwartz et al., 2005a, 2005b; Nishi et al., 2007) and in vivo (Bonny et al., 2004; Deo et al., 2006; Mihai et al., 2008).

In the present study, we examined the relationship between impact speed and injury severity using a custom-fabricated electromagnetically driven impact device. The accuracy of impact displacement was examined on a phantom and the mouse cord. The various injury severity levels were generated by fixing the displacement at $0.8 \mathrm{~mm}$ with three speficified impact speeds $(0.1,0.2$, and $0.4 \mathrm{~m} / \mathrm{s})$. The injury severity was evaluated by assessing the daily hind limb motor function using the Basso mouse scale (BMS) (Basso et al., 2006), and the extent of residual white matter using both in vivo diffusion tensor imaging (DTI) at 7 days post-injury (DPI) and the postmortem Luxol fast blue (LFB) at 21 DPI. The in vivo DTI (7DPI) and LFB (21 DPI) determined residual white matter extent consistently reflected in the BMS scores. The injury severity assessed by the extent of residual white matter or BMS scores was not different for the three tested impact speeds.

\section{Materials and Methods}

\section{Electromagnetic driven impactor}

The custom-built impactor is shown in Figure 1. The force of impact is provided by an electromagnetic actuator (Fig. 1D) (Model LA15-16-024A, BEI-Kimco, Vista, CA). Current is provided to the actuator via a linear amplifier (BTA-28V-6A, Precision MicroDynamics Inc., Victoria, British Columbia, Canada). The trajectory is controlled by a plastic flexure, which restricts motion in all directions except the desired impact direction (Fig. 1E). The impactor is mounted on a precision $\mathrm{XYZ}$ table (ThorLabs PT3A, ThorLabs Ltd., Newton, NJ) (Fig. 1C) to provide fine adjustments of $<100 \mu \mathrm{m}$ for locating desired target displacement. The desired impact displacement (displacement of impactor tip [Fig. 1H]) is preset by digital micrometer (Starrett 02064104, Parker-Hannifin/Daedal Division, Parker Hannifin Corporation, Irwin, PA). The micrometer provides a hard mechanical stop to limit the displacement of the impactor tip. The actual displacement of the impactor tip is measured by a laser displacement transducer (LD-1605, MicroEpsilon, Ortenburg, Germany), which provides fast, accurate measurements of tissue deformation (specified bandwidth $>5000 \mathrm{~Hz}$ ).

The impactor is driven from a laptop PC using a Matlab program incorporating the Data Acquisition Toolbox (MathWorks, Natick, MA). An analog IO card (National Instruments DAQ Card 6062-E), which is capable of acquiring from all channels at $10 \mathrm{KHz}$ and providing analog output commands at the same rate, provides the interface between the PC and the impactor. A command (Fig. 1b, bold line) from the PC specifies the current provided by the linear amplifier, which in turn controls the force delivered by the linear actuator. "Phantom" data are acquired with three preset displacements $(0.3 \mathrm{~mm}, 0.6 \mathrm{~mm}$, and $0.9 \mathrm{~mm})$ in both upward and downward directions (Fig. 1b).

\section{Impact procedures}

The basic impact procedure is summarized with a schematic illustration and the measured displacement at different speeds (Fig. 2). After laminectomy, the mouse spine was supported in a custom-designed holder (Fig. 1I) and placed on a stage (Fig. 1B). The impactor tip is placed on top of the exposed cord by adjustment of the XYZ table (Fig. 1C). To determine the touch point, a conductivity sensor was used (Brody et al., 2007), with microscopic validation (Fig. 2, phase I). After finding the zero position, the desired impact displacement was set by a digital micrometer (Fig. 1F). Impact was initiated by a bipolar command voltage signal (Fig. 2, bold solid line), amplified to a proportional current to the linear actuator (Fig. 1D). The impactor tip was driven by the generated electromagnetic force. The bipolar command first moved the impactor tip upward (Fig. 2, phase II) and then rapidly downward (Fig. 2, phase III). The downward movement of impactor tip was stopped at the desired impact displacement by the modified digital micrometer (Fig. 1F). After $\sim 20 \mathrm{~ms}$, the impactor tip was withdrawn above the initial position to avoid secondary impact (Fig. 2, phase IV). Both command voltage and impact displacement were set and acquired using a laptop computer through the analog IO card as in the phantom measurement.

\section{Spinal cord injury with various impact speeds}

All surgical interventions and both pre- and post-surgical care were performed in accordance with the Public Health Service Policy on Humane Care and Use of Laboratory Animals, Guide for the Care and Use of Laboratory Animals (Institute of Laboratory Animal Resources, National Research Council, 1996), and with the approval of the Washington University Institutional Care and Use Committee.

Twenty-four 10-week-old female C57BL/6 mice, weighing $18 \sim 20 \mathrm{~g}$ (Harlan, Indianapolis, IN), were anesthetized with an isoflurane and oxygen mixture (5\% for induction and $1.5 \%$ for maintenance). After a dorsal laminectomy at the T8 and T9 vertebral level, mice received contusive spinal cord injuries. Six mice (sham) received anesthesia, laminectomy, and underwent all procedures, including contact to establish the reference position, but no impact. Three injury groups of mice underwent contusion injury at $0.1,0.2$, and $0.4 \mathrm{~m} / \mathrm{s}$ with $0.8 \mathrm{~mm}$ impact displacement using 1.3-mm diameter rounded tip. After impact, the site was closed in layers with 4-0 silk sutures followed by subcutaneous administration of Baytril $(2.5 \mathrm{mg} / \mathrm{kg})$ and lactated Ringers $(5 \mathrm{~mL})$. Standard postoperative care including bladder expression was provided in accordance to the manual of the Spinal Cord Injury Research Training Program, published by the Spinal Trauma and Repair Laboratories at Ohio State University (http:// medicine.osu.edu/sci/).

\section{Hind limb motor function evaluation}

All mice underwent hind limb motor function evaluation with the BMS scale (Basso et al., 2006). All procedures were 
a
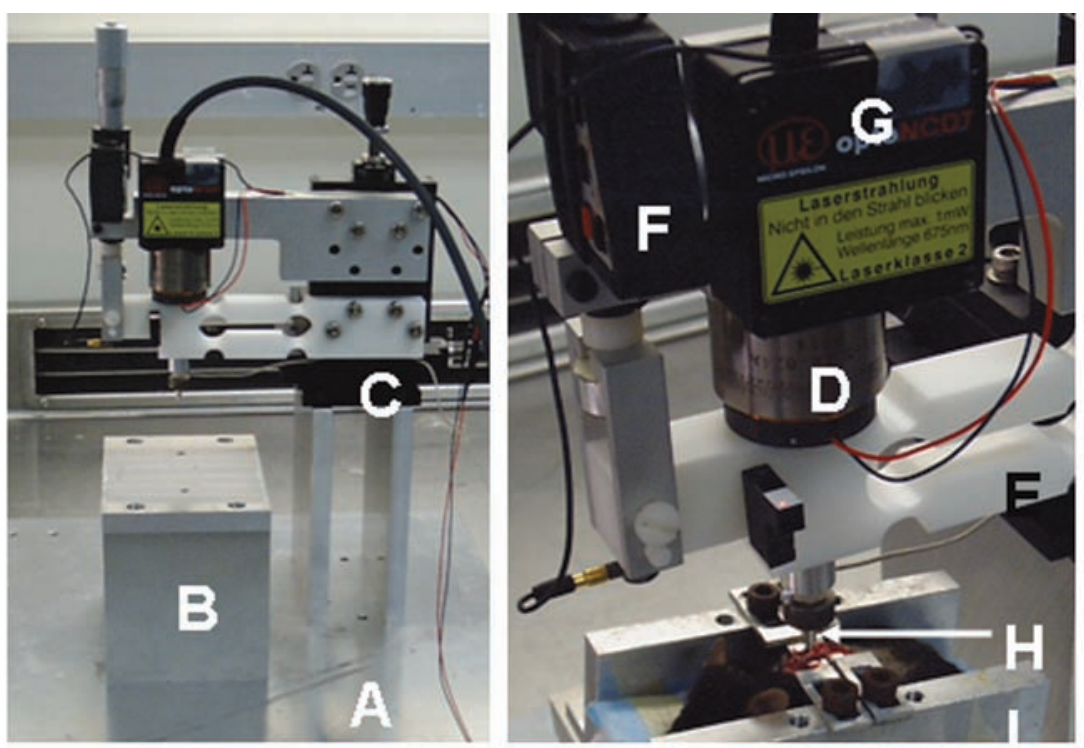

b

$\square: 0.3 \mathrm{~mm}, \Delta: 0.6 \mathrm{~mm}, \quad O: 0.9 \mathrm{~mm}(\mathrm{n}=10$ for each group)

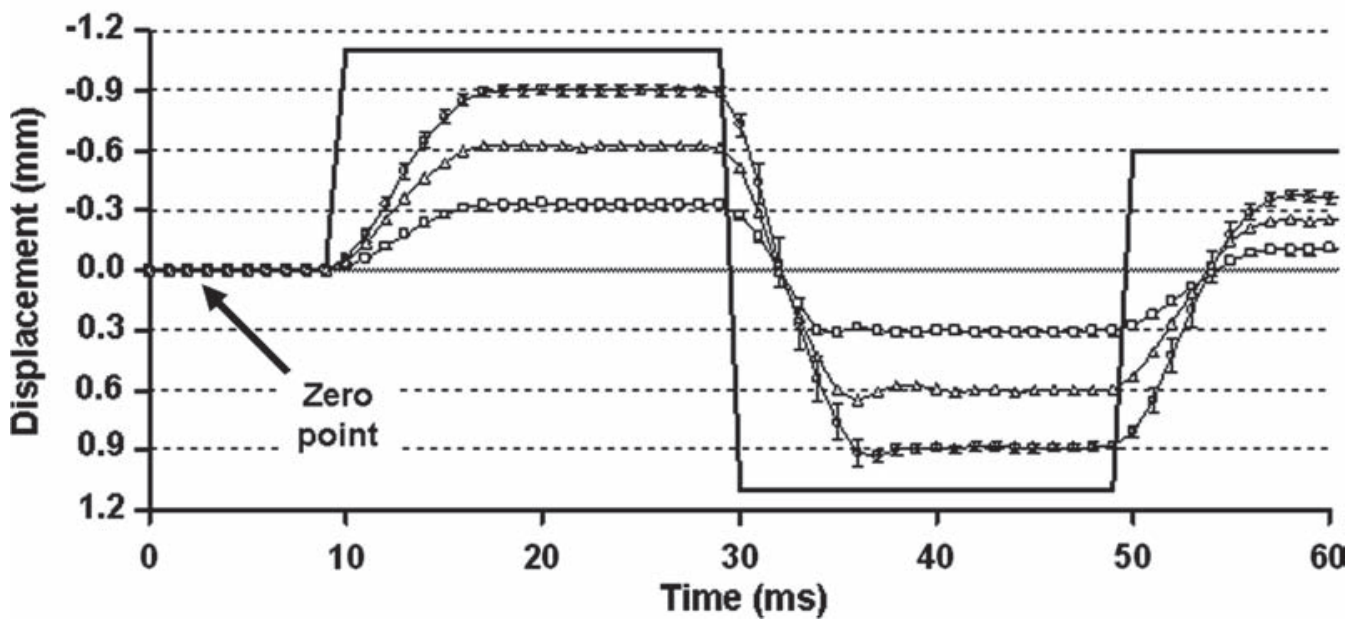

FIG. 1. The custom-built impact device (a). A, XY plate; B, stage for mouse; C, XYZ translator; D, linear actuator (provides electromagnetic force); E, flexure (restricts motion so impactor tip moves only at the desired z-direction); F, digital micrometer (used to set the impact distance); G, laser displacement transducer (measures the impact distance, i.e., displacement of the impactor tip); $\mathrm{H}$, impactor tip (13 mm diameter); I, mouse holder. (b) Measured impact displacements during phantom impacts $(\mathrm{n}=10)$ at various impact depths: $0.3 \mathrm{~mm}(\square), 0.6 \mathrm{~mm}(\triangle)$, and $0.9 \mathrm{~mm}(\bigcirc)$. Data are expressed as mean \pm standard deviation. Since the impact is conducted from the dorsal to ventral direction, a negative sign represents upward motion. The solid, bold line represents the command pulse (voltage, not related to the y-axis units). The zero point is indicated with an arrow. These displacement profiles during phantom impacts show high correspondence with desired trajectories, at impact depths from 0.3 to $0.9 \mathrm{~mm}$, for both upward and downward motion.

performed following the manual of the Spinal Cord Injury Research Training Program. Briefly, two raters blinded to the experimental conditions observed hind limb motor function of contusion-injured mice from 1 to 21 DPI. The rating was done daily during the first week. Mice were rated every other day during the second week, followed by ratings every 3 days in the third week. The worse deficit score was taken if two rater's scores did not match. At each time point, BMS score were averaged for each study group.

\section{Animal preparation for in vivo $D T I$}

At 7DPI, mice were delivered to MR facility and immobilized with an isoflurane and oxygen mixture $(1.5 \%$ for maintenance). Core body temperatures were maintained at $37^{\circ} \mathrm{C}$ with a circulating warm water pad. Inhaled isofluraneoxygen mixture was delivered to mice through a custommade nose cone. The respiratory exhaust line was connected to a pressure transducer to synchronize DTI data acquisition with the animal's respiratory rate (Kim et al., 2006). An MRIcompatible restraining device was utilized to stabilize the vertebral column as reported previously (Kim et al., 2006). An inductively coupled surface coil covering T8-T10 vertebral segments $(15 \mathrm{~mm} \times 8 \mathrm{~mm})$ was used as the RF receiver. A 9-cm i.d. Helmholtz coil was employed as the RF transmitter. The entire preparation was placed in an Oxford Instruments 200/330 magnet (4.7 T, 33-cm clear bore) equipped with a $15-\mathrm{cm}$ inner diameter, actively shielded Oxford gradient coil 


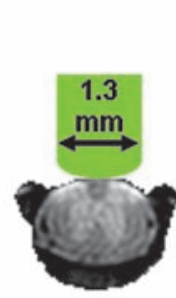

Phase I

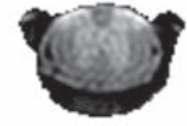

Phase II

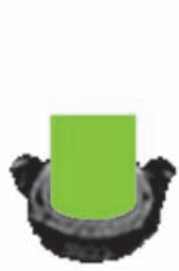

Phase III

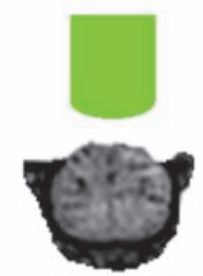

Phase IV

$\square: 0.1 \mathrm{~m} / \mathrm{s}, \triangle: 0.2 \mathrm{~m} / \mathrm{s}, \mathrm{O}: 0.4 \mathrm{~m} / \mathrm{s}$ (mean \pm SD. $n=6$ for each group)

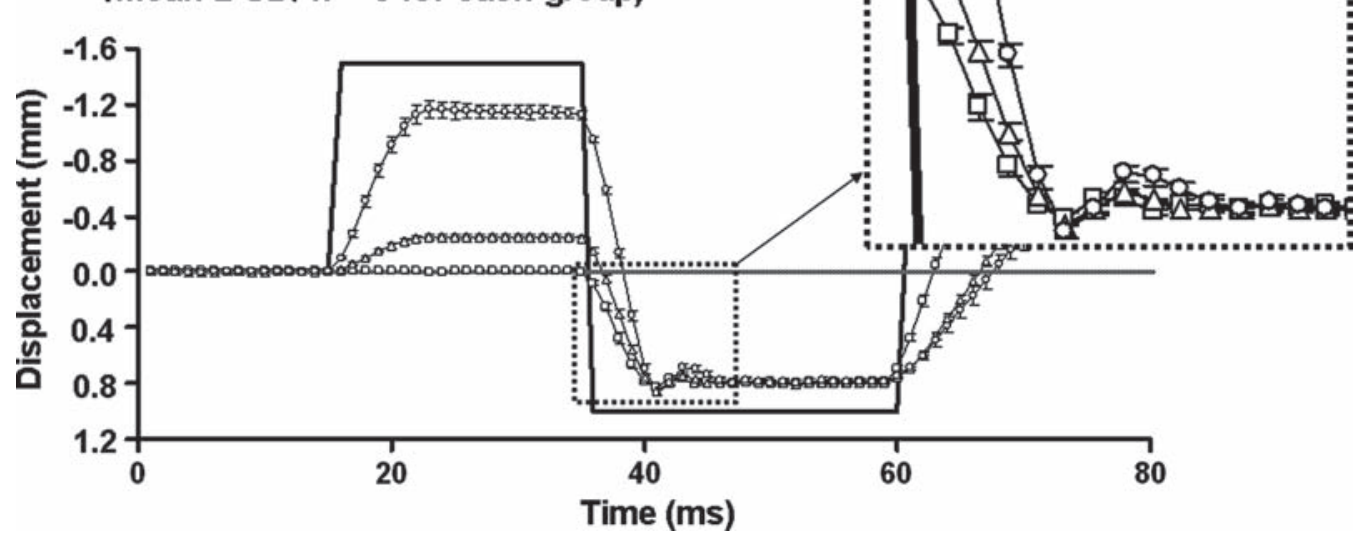

FIG. 2. The impact procedure on the mouse spinal cord is shown, along with a plot of the position of the impactor tip versus time. The bold, solid line represents the command pulse, in voltage (the amplitude is not to scale), and the displacement for $0.1 \mathrm{~m} / \mathrm{s}(\square), 0.2 \mathrm{~m} / \mathrm{s}(\triangle)$, and $0.4 \mathrm{~m} / \mathrm{s}(\bigcirc)$ are presented as mean \pm standard deviation $(\mathrm{n}=6$ for each group). Negative displacements represent the upward motion (from ventral to dorsal). Prior to phase I, the impactor tip was placed directly on top of the exposed cord by the XYZ translator. With the initiation of impact procedure via a computer command, the impactor tip was moved up (phase II) and rapidly down (impact occurs, phase III). The impactor tip was immediately moved up to avoid secondary impact.

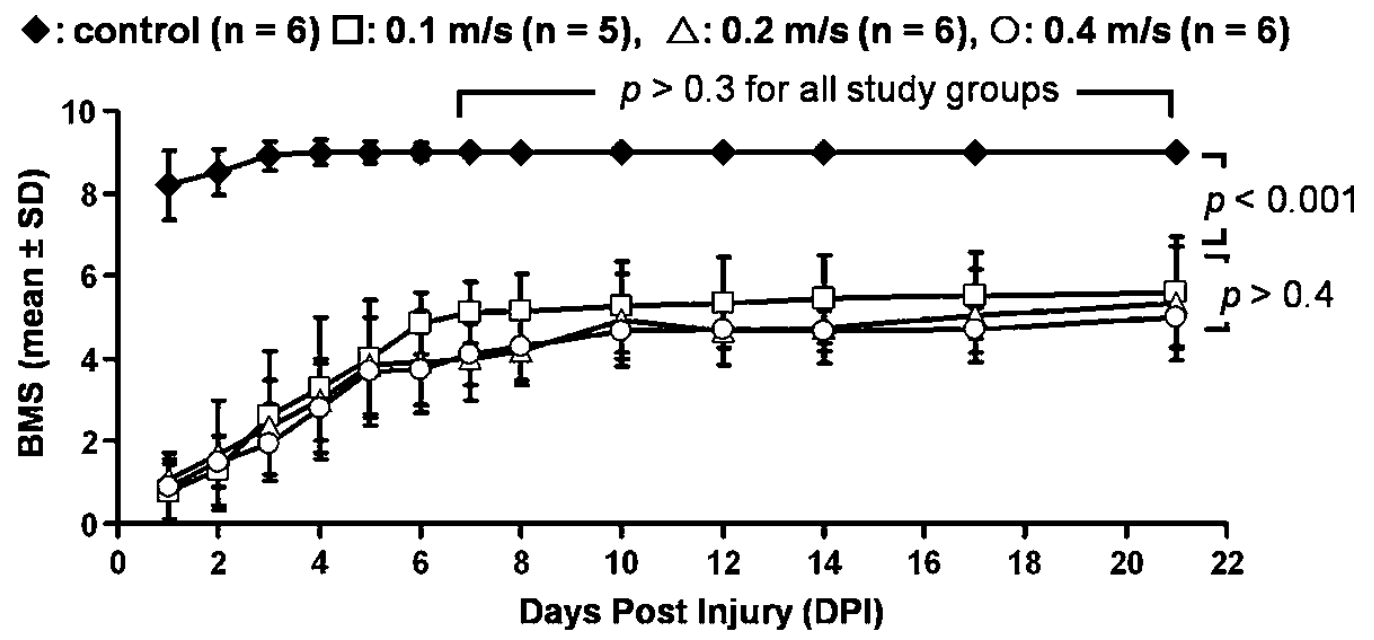

FIG. 3. Longitudinal Basso mouse scale (BMS) for control $(\bullet)$ and impacts at $0.1 \mathrm{~m} / \mathrm{s}(\square), 0.2 \mathrm{~m} / \mathrm{s}(\triangle)$, and $0.4 \mathrm{~m} / \mathrm{s}(\bigcirc)$. Results are presented as mean \pm standard deviation. All groups recovered from the initial severe functional deficit reaching a steady-state BMS score ( $5 \pm 0.8)$ after 7 DPI. No significant functional differences among injury groups were observed where all injury groups have significantly lower scores than the control. 
a

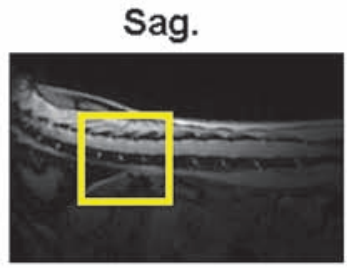

Cor. 1
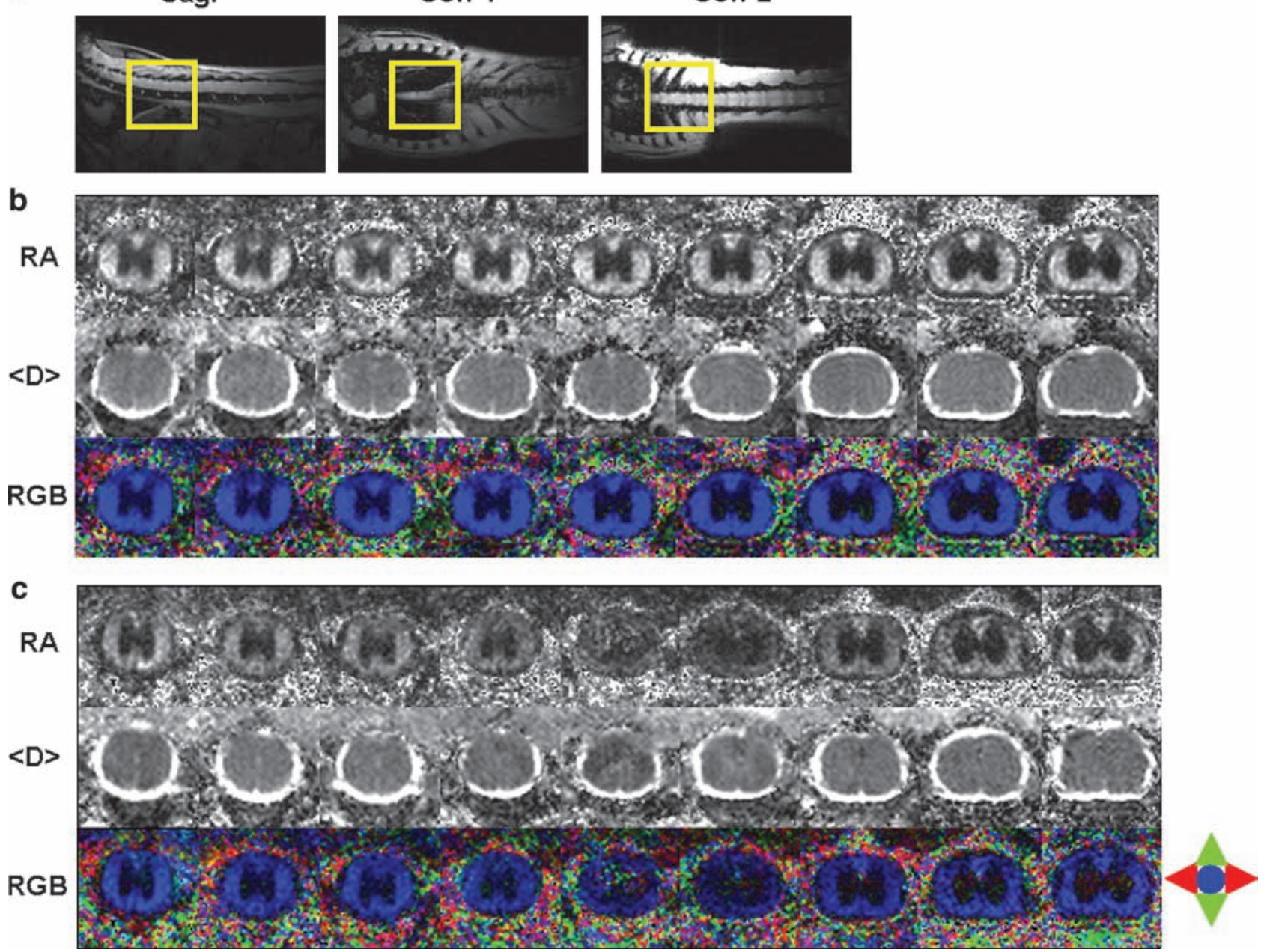

FIG. 4. (a) Scout sagittal and coronal images, (b) serial transverse in vivo diffusion tensor images of the control, and (c) injured cords are demonstrated. Nine contiguous transverse (or axial) images were acquired encompassing T7-T10 vertebrae level (voxel size $=39 \mu \mathrm{m} \times 39 \mu \mathrm{m}$ (zero-filled); slice thickness $=1 \mathrm{~mm}$ ). Relative anisotropy (RA) and apparent mean diffusivity $(\langle\mathrm{D}\rangle)$ are displayed in scale of $0-1.414$ and $0-2 \mu \mathrm{m}^{2} / \mathrm{ms}$. The parenchyma of the spinal cord is hypointense compared to the surrounding bright CSF in the $<\mathrm{D}>$ map. The gray-white matter contrast is clearly seen in the RA map. The coherent directionality of white matter is obvious in the color-coded RA map where red is left to right, green is up and down, and blue is in and out of the image plane. The epicenter of the injury is easily identified from the RA map.

(18G/cm, 200- $\mu$ s rise time). The magnet, gradient coil, and Techron gradient power supply were interfaced with a Varian UNITY-INOVA console (Palo Alto, CA) controlled by a Sun Microsystems Blade 1500 workstation.

\section{In vivo diffusion tensor imaging}

A conventional spin-echo imaging sequence was modified by adding Stejskal-Tanner diffusion weighting gradients (Stejskal, 1965). The spin echo time (TE $=38 \mathrm{~ms})$, time between application of gradient pulses $(\Delta=20 \mathrm{~ms})$, and diffusion gradient on time $(\delta=7 \mathrm{~ms})$ were fixed throughout the experiment. The repetition time (TR $\sim 1.2 \mathrm{~s}$ ) was varied according to the period of the respiratory cycle $(\sim 270 \mathrm{~ms})$. During every breath, three different image slices were collected. Images were obtained with diffusion sensitizing gradients applied in six orientations: $(\mathrm{Gx}, \mathrm{Gy}, \mathrm{Gz})=(1,1,0)$,
$(1,0,1),(0,1,1),(-1,1,0]),(0,-1,1)$, and $(1,0,-1)$. Two diffusionsensitizing factors ( $b$ values) of 0 and $1.0 \mathrm{~ms} / \mu \mathrm{m}^{2}$ were used. Eight scans were averaged per k-space line. The field of view was $10 \times 10 \mathrm{~mm}^{2}$ with $1.0 \mathrm{~mm}$ slice thickness, and the image data matrix for each slice was $128(\mathrm{PE}) \times 256(\mathrm{RO})$ (zero filled to $256 \times 256$ ).

\section{Data analyses}

Diffusion tensors were estimated independently for each voxel from the diffusion-weighted images using a weighted linear least-squares method (Koay et al., 2006). The eigenvalue decomposition was then applied to each tensor, yielding a set of eigenvalues $\left(\lambda_{1} \geq \lambda_{2} \geq \lambda_{3}\right)$ and eigenvectors for each voxel. Maps of diffusion indices, including relative anisotropy (RA) and apparent mean diffusivity $(<\mathrm{D}>)$, were generated by applying the following equations for each voxel. 

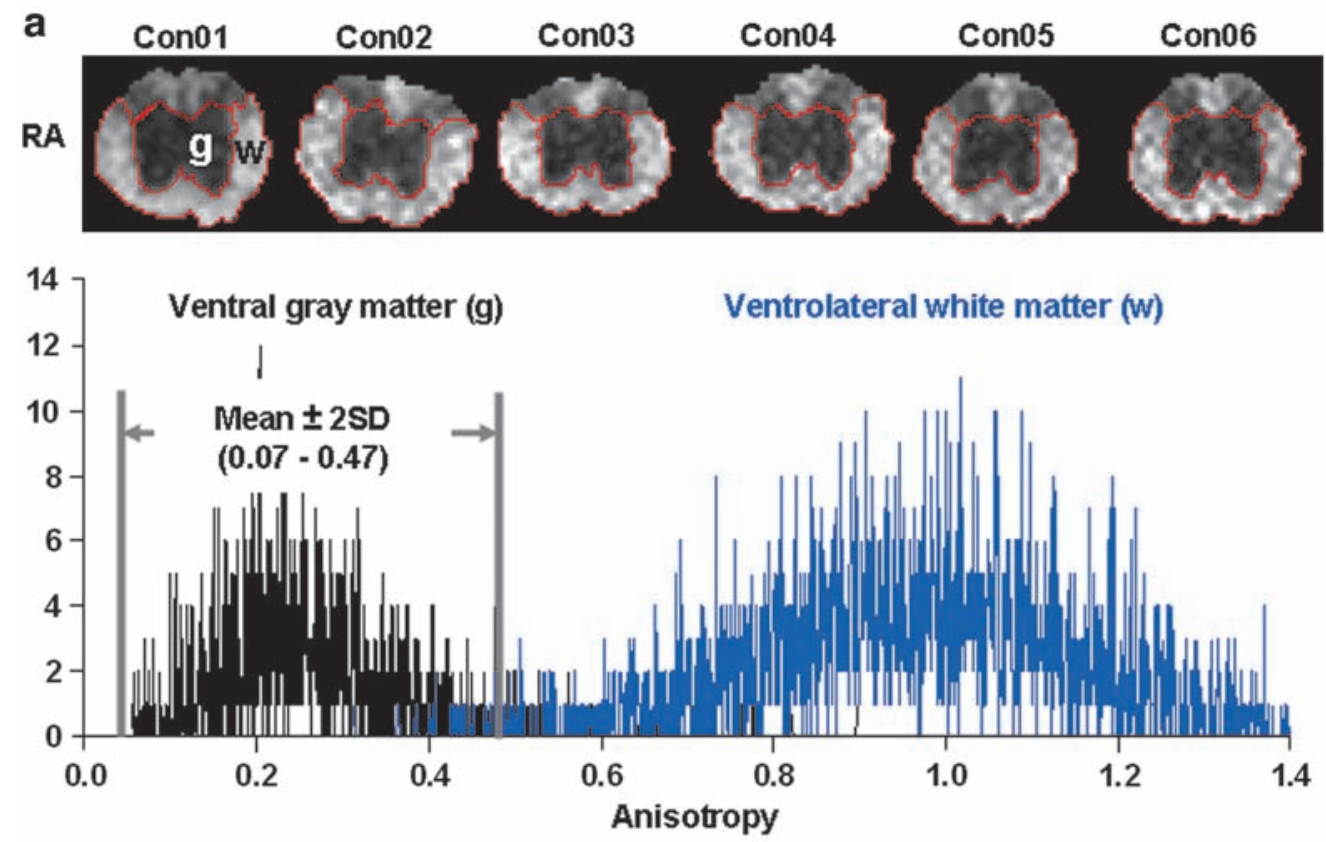

b
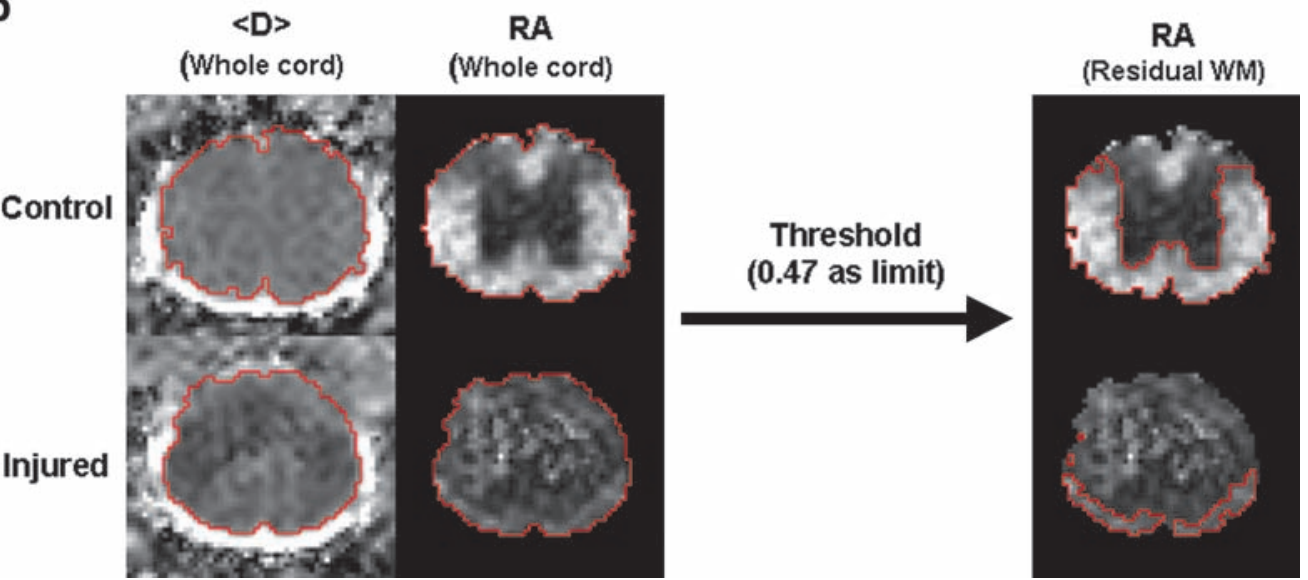

FIG. 5. (a) The anisotropy distribution of ventral gray matter (VGM, g) and ventrolateral white matter (VLWM, w) from six control mice (Con01-Con06) are shown with dorsal gray and white matter excluded. Two normally distributed RA values are clearly seen from VGM $(0.27 \pm 0.10)$ and VLWM $(0.97 \pm 0.19)$. All data are presented as mean \pm standard deviation. A threshold of $\mathrm{RA}=$ mean $+2 \times$ standard deviation (i.e., 0.47 ) of VGM was employed to successfully separate the ventrolateral white matter from the gray matter $(\mathbf{b})$.

$$
\begin{gathered}
<\mathrm{D}>=\left(\lambda_{1}+\lambda_{2}+\lambda_{3}\right) / 3 \\
\mathrm{RA}=\frac{\sqrt{\left(\lambda_{1}-<\mathrm{D}>\right)^{2}+\left(\lambda_{2}-<\mathrm{D}>\right)^{2}+\left(\lambda_{3}-<\mathrm{D}>\right)^{2}}}{\sqrt{3}<\mathrm{D}>}
\end{gathered}
$$

The RA map was additionally color coded to include information regarding the primary diffusion direction (i.e., the direction of the principle eigenvector). Specifically, the colors blue, red, and green correspond to rostrocaudal, mediolateral, and superoinferior diffusion directions, respectively, with the brightness of the color maps weighted by the RA (Pajevic and Pierpaoli, 1999).

To quantify the diffusion parameters, a region-of-interest (ROI) analysis was conducted. In control cords, the manual segmentation of ventrolateral white matter (VLWM) and ventral gray matter (VGM) was performed based on RA maps and the non-diffusion weighted image $\left(b=0 \mathrm{~ms} / \mu \mathrm{m}^{2}\right)$. At T9 of control cords $(n=6)$, the RA histogram of VGM and VLWM were generated from manually segmented ROI producing mean and standard deviation (SD) for each VGM and VLWM, based on the histogram of the distribution of the control VGM and VLWM. The normal appearing white matter in VLWM region was determined by employing a threshold of mean $+2 \times$ standard deviation of RA in the control VGM.

\section{Tissue preparation and histological analysis}

At the conclusion of longitudinal hind limb motor function examinations, all mice were perfusion fixed through the left cardiac ventricle under deep anesthesia with $4 \%$ paraformaldehyde in phosphate-buffered saline (PBS, pH 7.4). Fol- 
a

In vivo RA maps at 7 DPI

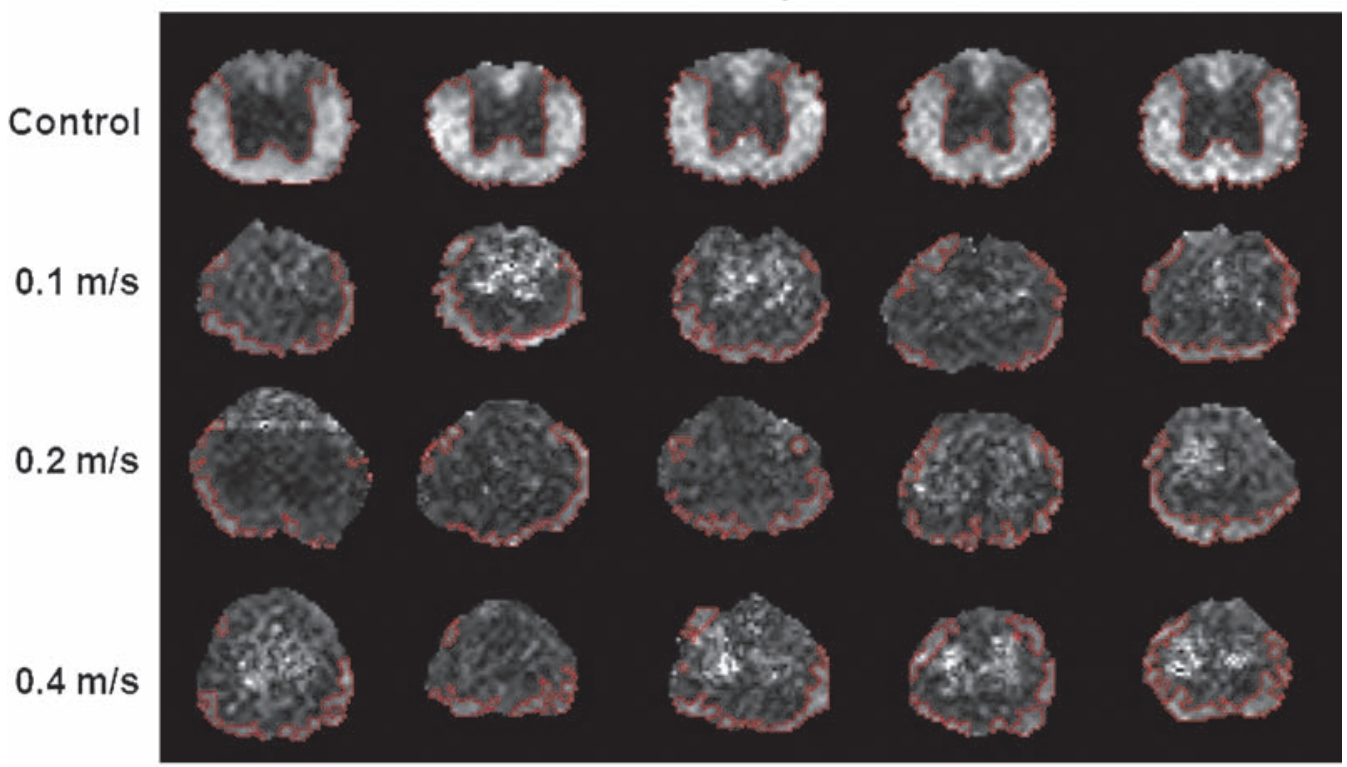

b Control $(n=6), 0.1 \mathrm{~m} / \mathrm{s}(n=5), 0.2 \mathrm{~m} / \mathrm{s}(n=6), 0.4 \mathrm{~m} / \mathrm{s}(n=6)$

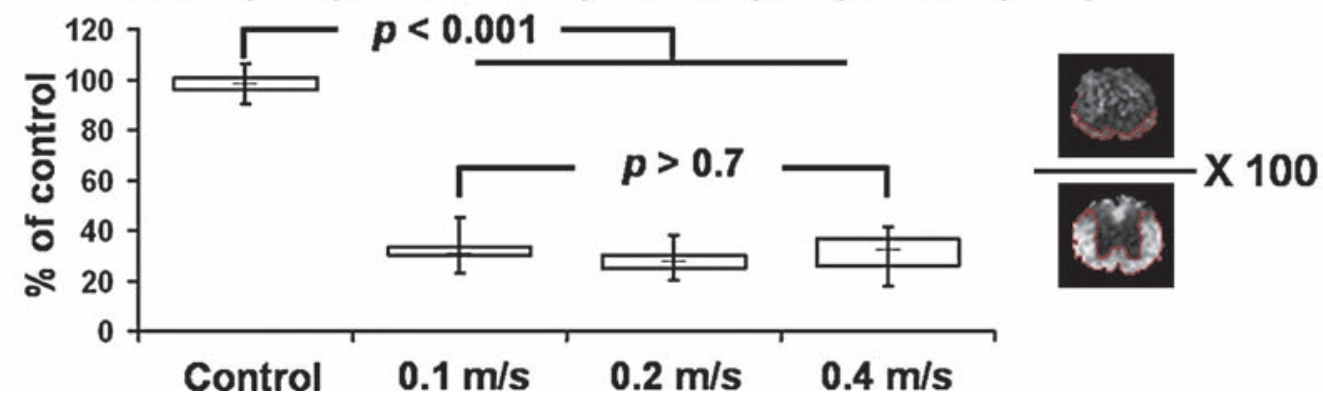

FIG. 6. (a) The normal-appearing residual VLWM determined using the RA threshold (Fig. 5) at the epicenter from five individual mice. Control cords have ROIs containing entire ventrolateral white matter. All injured cords show normalappearing residual white matter mostly at the outer rim of the parenchyma. (b) The normal-appearing VLWM volume, normalized to the control, for control $(n=6), 0.1 \mathrm{~m} / \mathrm{s}(\mathrm{n}=5), 0.2 \mathrm{~m} / \mathrm{s}(\mathrm{n}=6)$, and $0.4 \mathrm{~m} / \mathrm{s}(\mathrm{n}=6)$ groups at 7 DPI were obtained. All injured groups show a significant decrease in the normal-appearing VLWM volume compared with that of the control. There is no statistically significant difference among all injured groups.

lowing fixation, the spine was harvested, left in fixative for $24 \mathrm{~h}$, and then decalcified. A 2-mm segment of spinal cord centered at T9 was cut with the vertebral column intact after decalcification. Spinal cord blocks were embedded in paraffin and cut on a sliding microtome at a thickness of $3 \mu \mathrm{m}$. For the examination of myelin integrity, luxol fast blue (LFB) staining was conducted. Briefly, the sectioned tissue underwent deparaffinization and rehydration. After overnight immersion in LFB solution $\left(56^{\circ} \mathrm{C}\right)$, excess stain was rinsed off and differentiation was performed with lithium carbonate solution and ethyl alcohol. After the completion of differentiation, tissue was mounted for microscopic inspection. Spared residual white matter volume for control and injured cord was evaluated utilizing ImageJ 1.37 (Rasband, 1997-2005).

\section{Statistical analysis}

Repeated measures ANOVA was employed to assess the difference of animal's hind limb motor function using SAS, version 9.1.3 (SAS Institute, Cary, NC), with Tukey as post hoc test. The statistical significance was accepted as $p<0.05$. At each time point, the group averaged BMS scores of the three injury groups were compared. The longitudinal variance of BMS within each study group including control was tested. One-way ANOVA was employed to examine the difference of spared white matter volume determined by subacute in vivo RA and end point LFB. The significance of difference between control and injury groups, and among the three injury groups was tested. The in vivo RA threshold segmented normal appearing residual white matter volume of the injured cord was normalized to the averaged normalappearing white matter volume of control cords $(n=6)$. The spared residual white matter volume determined by LFB was normalized to those of the control.

\section{Results}

The measured impact displacement (displacement of impactor tip) in "phantom" experiments and in the mouse spinal cord is shown as a function of time in Figures $1 b$ and 2 . In both 
phantom and cord experiments, the downward speed was greater than the upward (negative sign). The overshoot $(\sim 0.02 \mathrm{~mm})$, occurring in the fast downward movement, was insignificant compared to the desired impact displacement, preset by digital micrometer at $0.3,0.6$, and $0.9 \mathrm{~mm}$ (Fig. 1b). From contusion injured cord, consistent displacement $(0.8 \mathrm{~mm})$ and the regulated speed (from $0.1,0.2$, and $0.4 \mathrm{~m} / \mathrm{s}$ ) were observed with same duration at the maximum displacement (Fig. 2). The overshoot and undershoot of the impact displacement on mouse cord are also negligible compared to desired displacement at the speed ranging from 0.1 to $0.4 \mathrm{~m} / \mathrm{s}$.

Longitudinal hind limb motor function was evaluated (Fig. 3). All mice recovered from initial surgery and spinal shock during the first week after injury. The BMS score of all injury groups were significantly lower than that of the control longitudinally $\left(F_{3,36}=698.3, p<0.001\right)$. After $7 \mathrm{DPI}$, the BMS score reached steady state showing no statistically significant difference from 7 to 21 DPI for all groups $(p>0.3)$. No statistically significant difference in steady state BMS score was observed among three injury groups of different impact speeds $(p>0.4)$.
The serial in vivo DTI maps of the control and contusion injured cord at 7 DPI were localized based on both sagittal and coronal scout images (Fig. 4a). Gray scale and color-coded RA maps showed clear gray-white matter contrast (Fig. 4b) even at the epicenter of the injured cord (Fig. 4c). The coherent blue color of the white matter was consistent with the structure of the myelinated axons (Fig 4b). The epicenter was easily identified through the significantly decreased RA (Fig. 4c).

An objective evaluation of the injury severity based on the normal-appearing VLWM was performed using RA threshold (Fig. 5). This RA threshold (0.47) was applied to the epicenter of all injured cords at 7 DPI to determine the extent of the normal-appearing VLWM volume (Fig. 6a). The normalappearing VLWM extent of contusion injured cord was normalized to the averaged normal VLWM volume of control cords (Fig. 6b). There was no statistically significant difference in the spared VLWM among all injury groups $\left(\mathrm{F}_{2,15}=0.0399\right.$, $p>0.7)$, where there was a significant reduction from control $\left(\mathrm{F}_{3,20}=126.82, p<0.001\right.$; Fig. $\left.6 \mathrm{~b}\right)$.

At the end of longitudinal evaluation of BMS scores (21 DPI), the residual white matter at epicenter was assessed with LFB (Fig. 7a). Residual white matter was quantified by
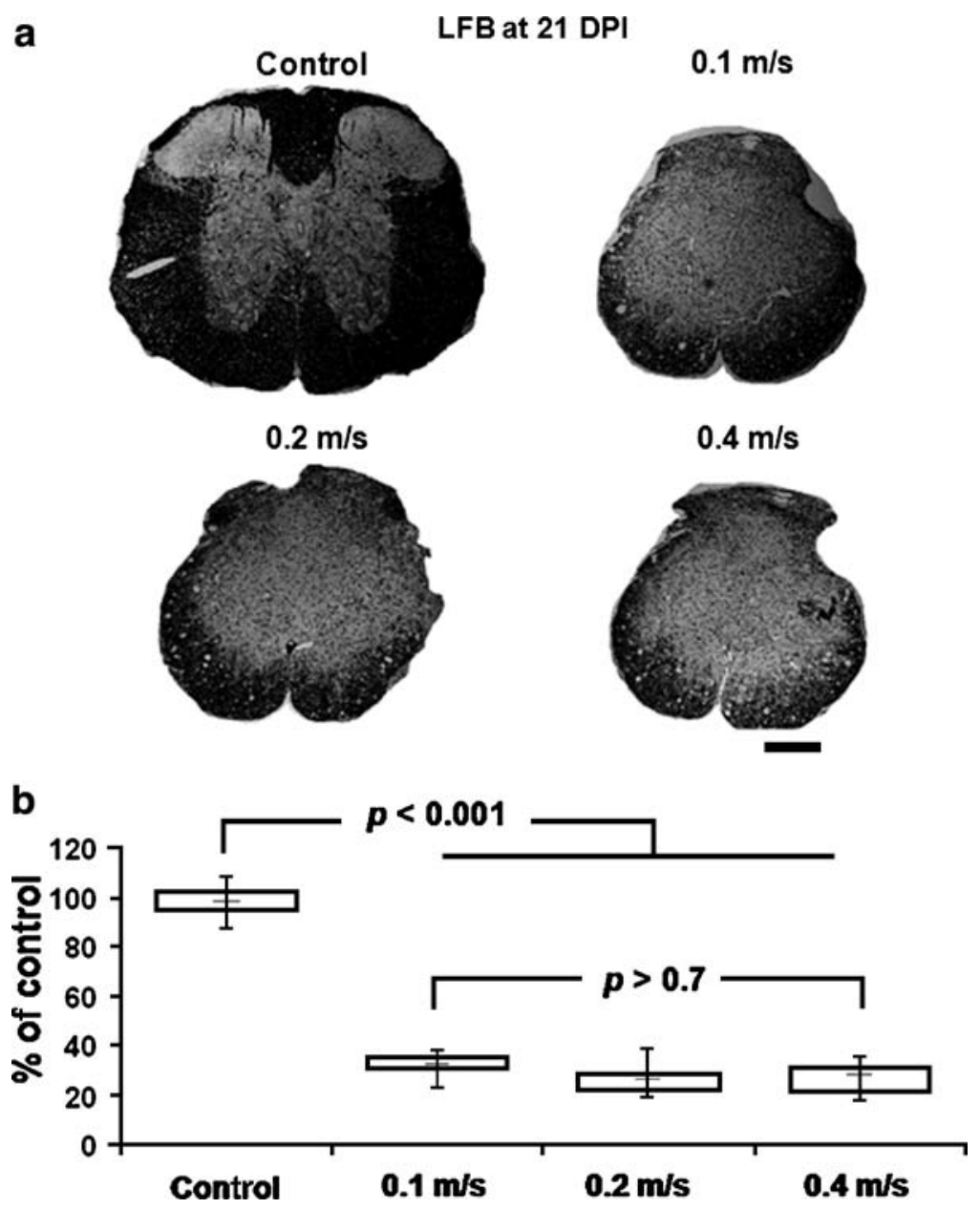

FIG. 7. Luxol fast blue (LFB) staining at the epicenter of injury (a), and the residual VLWM volume at 21 DPI for control $(\mathrm{n}=6)$, and injury groups of impact speed at $0.1 \mathrm{~m} / \mathrm{s}(\mathrm{n}=5), 0.2 \mathrm{~m} / \mathrm{s}(\mathrm{n}=6)$, and $0.4 \mathrm{~m} / \mathrm{s}(\mathrm{n}=6)(\mathrm{b})$. The spared residual white matter volume of the injured cord is normalized by the averaged volume of control cords. The residual white matter of the injured cords is significantly smaller than the control, but there is no statistically significant difference among injured cords. Scale bar, $100 \mu \mathrm{m}$. 
normalizing to the averaged total white matter volume from the control mice. Similar to the MRI findings at $7 \mathrm{DPI}$, there was no statistically significant difference in the residual white matter contents, determined using histology, among all injury groups at $21 \mathrm{DPI}\left(\mathrm{F}_{2,15}=0.2690, p>0.7\right)$. However, there was a significant reduction in spared VLWM in the injury groups comparing with the control $\left(\mathrm{F}_{3,20}=159.85, p<0.001\right.$; Fig. 7b).

\section{Discussion}

In this study, we examined the relationship between impact speed and injury severity in a mouse model of contusion SCI. Using a custom-built device that was set to a fixed impact displacement $(0.8 \mathrm{~mm})$, impact speed was varied over a range covering the three major rodent SCI devices (IH: $0.1 \mathrm{~m} / \mathrm{s}$, OSU: $0.2 \mathrm{~m} / \mathrm{s}$, and NYU: $0.4 \mathrm{~m} / \mathrm{s}$ ). Multiple outcome measures (hind limb motor function assessed with BMS, normalappearing white matter determined using in vivo DTI, and post-mortem spared white matter estimated by LFB) were evaluated at various time points (longitudinal BMS, in vivo DTI at 7 DPI, and LFB at 21 DPI). The current findings show no statistically significant correlation between impact speed or injury severity at this fixed displacement.

The ability to control and reproduce different injury severities is crucial to evaluate treatment efficacy, as well as of the underlying mechanisms, of the injury. In this study, we regulated displacement and velocity using a custom-fabricated impactor. The displacement and trajectory were controlled using a flexure-based design (Fig. 1A-E). The extent of overshoot was reduced with micrometer-control and a rigid mechanical stop specifying impact depth. Insignificant overshoots $(\sim 0.02 \mathrm{~mm})$ were observed in this study at a speed up to $0.4 \mathrm{~m} / \mathrm{s}$ (Fig. 2).

The hind limb motor function has been widely employed to assess injury severity (Basso et al., 1995, 1996, 2006; EngesserCesar et al., 2005; Magnuson et al., 2005) and the efficacy of treatments in rodent models of SCI (Engesser-Cesar et al., 2005; Steward et al., 2008). The longitudinal BMS suggested no significant difference in the hind limb motor function among the three injury groups of different impact speed. Even though behavioral tests are reliable and widely employed, it lacks the crucial localization information of the injury. DTI is a noninvasive modality that can provide quantitative assessments of SCI progression in rodents (Deo et al., 2006; Kim et al., 2007; Loy et al., 2007).

In vivo DTI was performed in mice at $7 \mathrm{DPI}$, coinciding with the steady state of hind limb motor function assessed with BMS (Fig. 3). The normal-appearing VLWM at the epicenter was quantified since the hind limb motor function after injury would be strongly affected by the degree of white matter preservation at the epicenter (Kuhn and Wrathall, 1998; Ma et al., 2001; Nishi et al., 2007; Plemel et al., 2008). All injured cords had significantly reduced normal-appearing VLWM volume compared with that of the control. However, all three injury groups showed similar normal-appearing VLWM volume at epicenter regardless of the impacting speed.

The spared white matter was also assessed with postmortem LFB at the conclusion of the course of the study. Similar to in vivo RA findings, the comparable extent of spared white matter was observed for all three injury groups. Even though RA and LFB were not measured concurrently, both measurements agreed in the location and extent of spared residual white matter.

In conclusion, the current finding suggests that speed variations spanning the range of three widely employed rodent SCI impact devices (IH: $0.1 \mathrm{~m} / \mathrm{s}$, OSU: $0.2 \mathrm{~m} / \mathrm{s}$, and NYU: $0.4 \mathrm{~m} / \mathrm{s}$ ) do not affect injury severity if the displacement is controlled. In addition, estimates of spared white matter obtained using in vivo DTI derived RA maps compare closely with estimates from post-mortem LFB, suggesting that in vivo DTI can be used to noninvasively measure white matter injury in the contusion injured spinal cord.

\section{Acknowledgments}

This study was supported in part by the National Institute of Health (NS047592), and the University of Missouri Spinal Cord Injuries Research Program. The authors thank Jian Wang for the insightful discussion and performing statistical analyses.

\section{Author Disclosure Statement}

No competing financial interest exist.

\section{References}

Basso, D.M., Beattie, M.S., and Bresnahan, J.C. (1995). A sensitive and reliable locomotor rating scale for open field testing in rats. J. Neurotrauma 12, 1-21.

Basso, D.M., Beattie, M.S., and Bresnahan, J.C. (1996). Graded histological and locomotor outcomes after spinal cord contusion using the NYU weight-drop device versus transection. Exp. Neurol. 139, 244-256.

Basso, D.M., Fisher, L.C., Anderson, A.J., Jakeman, L.B., McTigue, D.M., and Popovich, P.G. (2006). Basso Mouse Scale for locomotion detects differences in recovery after spinal cord injury in five common mouse strains. J. Neurotrauma 23, 635-659.

Bonny, J.M., Gaviria, M., Donnat, J.P., Jean, B., Privat, A., and Renou, J.P. (2004). Nuclear magnetic resonance microimaging of mouse spinal cord in vivo. Neurobiol. Dis. 15, 474-482.

Brody, D.L., Mac Donald, C., Kessens, C.C., Yuede, C., Parsadanian, M., Spinner, M., Kim, E., Schwetye, K.E., Holtzman, D.M., and Bayly, P.V. (2007). Electromagnetic controlled cortical impact device for precise, graded experimental traumatic brain injury. J. Neurotrauma 24, 657-673.

Deo, A.A., Grill, R.J., Hasan, K.M., and Narayana, P.A. (2006). In vivo serial diffusion tensor imaging of experimental spinal cord injury. J. Neurosci. Res. 83, 801-810.

Engesser-Cesar, C., Anderson, A.J., Basso, D.M., Edgerton, V.R., and Cotman, C.W. (2005). Voluntary wheel running improves recovery from a moderate spinal cord injury. J. Neurotrauma 22, 157-171.

Ghasemlou, N., Kerr, B.J., and David, S. (2005). Tissue displacement and impact force are important contributors to outcome after spinal cord contusion injury. Exp. Neurol. 196, 9-17.

Gruner, J.A. (1992). A monitored contusion model of spinal cord injury in the rat. J. Neurotrauma 9, 123-126; discussion 126128.

Jakeman, L.B., Guan, Z., Wei, P., Ponnappan, R., Dzwonczyk, R., Popovich, P.G., and Stokes, B.T. (2000). Traumatic spinal cord injury produced by controlled contusion in mouse. J. Neurotrauma 17, 299-319.

Kim, J.H., Budde, M.D., Liang, H.F., Klein, R.S., Russell, J.H., Cross, A.H., and Song, S.K. (2006). Detecting axon damage in 
spinal cord from a mouse model of multiple sclerosis. Neurobiol. Dis. 21, 626-632.

Kim, J.H., Loy, D.N., Liang, H.F., Trinkaus, K., Schmidt, R.E., and Song, S.K. (2007). Noninvasive diffusion tensor imaging of evolving white matter pathology in a mouse model of acute spinal cord injury. Magn. Reson. Med. 58, 253-260.

Koay, C.G., Chang, L.C., Carew, J.D., Pierpaoli, C., and Basser, P.J. (2006). A unifying theoretical and algorithmic framework for least squares methods of estimation in diffusion tensor imaging. J. Magn. Reson. 182, 115-125.

Kuhn, P.L., and Wrathall, J.R. (1998). A mouse model of graded contusive spinal cord injury. J. Neurotrauma 15, 125-140.

Loy, D.N., Kim, J.H., Xie, M., Schmidt, R.E., Trinkaus, K., and Song, S.K. (2007). Diffusion tensor imaging predicts hyperacute spinal cord injury severity. J. Neurotrauma 24, 979-990.

Ma, M., Basso, D.M., Walters, P., Stokes, B.T., and Jakeman, L.B. (2001). Behavioral and histological outcomes following graded spinal cord contusion injury in the $\mathrm{C} 57 \mathrm{Bl} / 6$ mouse. Exp. Neurol. 169, 239-254.

Magnuson, D.S., Lovett, R., Coffee, C., Gray, R., Han, Y., Zhang, Y.P., and Burke, D.A. (2005). Functional consequences of lumbar spinal cord contusion injuries in the adult rat. J. Neurotrauma 22, 529-543.

Mihai, G., Nout, Y.S., Tovar, C.A., Miller, B.A., Schmalbrock, P., Bresnahan, J.C., and Beattie, M.S. (2008). Longitudinal comparison of two severities of unilateral cervical spinal cord injury using magnetic resonance imaging in rats. J. Neurotrauma $25,1-18$.

Nevo, U., Hauben, E., Yoles, E., Agranov, E., Akselrod, S., Schwartz, M., and Neeman, M. (2001). Diffusion anisotropy MRI for quantitative assessment of recovery in injured rat spinal cord. Magn. Reson. Med. 45, 1-9.

Nishi, R.A., Liu, H., Chu, Y., Hamamura, M., Su, M.Y., Nalcioglu, O., and Anderson, A.J. (2007). Behavioral, histological, and ex vivo magnetic resonance imaging assessment of graded contusion spinal cord injury in mice. J. Neurotrauma 24, 674-689.

Pajevic, S., and Pierpaoli, C. (1999). Color schemes to represent the orientation of anisotropic tissues from diffusion tensor data: application to white matter fiber tract mapping in the human brain. Magn. Reson. Med. 42, 526-540.

Plemel, J.R., Duncan, G., Chen, K.W., Shannon, C., Park, S., Sparling, J.S., and Tetzlaff, W. (2008). A graded forceps crush spinal cord injury model in mice. J. Neurotrauma 25, 350-370.

Rasband, W.S. (1997-2005). ImageJ. U.S. National Institutes of Health, Bethesda, MD.
Scheff, S.W., Rabchevsky, A.G., Fugaccia, I., Main, J.A., and Lumpp, J.E., Jr. (2003). Experimental modeling of spinal cord injury: characterization of a force-defined injury device. J. Neurotrauma 20, 179-193.

Schwartz, E.D., Chin, C.L., Shumsky, J.S., Jawad, A.F., Brown, B.K., Wehrli, S., Tessler, A., Murray, M., and Hackney, D.B. (2005a). Apparent diffusion coefficients in spinal cord transplants and surrounding white matter correlate with degree of axonal dieback after injury in rats. Am. J. Neuroradiol. 26, 7-18.

Schwartz, E.D., Cooper, E.T., Fan, Y., Jawad, A.F., Chin, C.L., Nissanov, J., and Hackney, D.B. (2005b). MRI diffusion coefficients in spinal cord correlate with axon morphometry. Neuroreport 16, 73-76.

Schwartz, E.D., and Hackney, D.B. (2003). Diffusion-weighted MRI and the evaluation of spinal cord axonal integrity following injury and treatment. Exp. Neurol. 184, 570-589.

Stejskal, E., and Tanner, J. E. (1965). Spin echoes in the presence of a time-dependent field gradient. J. Chem. Phys. 42(1), 288-292.

Steward, O., Sharp, K., Yee, K.M., and Hofstadter, M. (2008). A re-assessment of the effects of a Nogo-66 receptor antagonist on regenerative growth of axons and locomotor recovery after spinal cord injury in mice. Exp. Neurol. 209, 446-468.

Stokes, B.T. (1992). Experimental spinal cord injury: a dynamic and verifiable injury device. J. Neurotrauma 9, 129-131; discussion 131-124.

Young, W. (2002). Spinal cord contusion models. Prog. Brain. Res. 137, 231-255.

Address correspondence to: Sheng-Kwei "Victor" Song, Ph.D. Washington University School of Medicine Campus Box 8227

4525 Scott Avenue, Room 2313

St. Louis, MO 63110

E-mail: ssong@wustl.edu

Philip V. Bayly, Ph.D.

Department of Mechanical and Aerospace Engineering

Washington University Campus Box 1185

1 Brookings Drive

St. Louis, MO 63130

E-mail: pvb@wustl.edu 